Ann. Sci. For., 1988, 45 (4), 357-381

\title{
Estimation des niveaux d'infestation passés de la tordeuse du sapin Choristoneura murinana (Hb.) (Lép., Tortricidae) par analyse rétrospective des pertes de feuillage et de croissance des arbres
}

\author{
P. DU MERLE, D. GÉRAUD * \\ avec la collaboration technique de E. ROBERT \\ INRA, Station de Zoologie forestière, Centre de Recherches d'Avignon, \\ avenue A. Vivaldi, F 84000 Avignon
}

\begin{abstract}
Summary
Estimating the past annual activity of the fir budworm Choristoneura murinana (Hb.) (Lep., Tortricidae) through retrospective analysis of the foliage and growth losses of the trees.

The budworm Choristoneura murinana has recently proved to severely damage silver fir (Abies alba Mill.) stands in the south of France. Understanding this situation, perhaps only new apparently, and assessing its implications would need to have informations on the past activity of the pest. Three criteria from trees likely to supply such data were tested: the needle loss of the shoots, the annual length increment of the branches and the annual radial increment of the trees. Observations were made in the departement of Ardèche, in two plots of a mature silver fir stand infested by the budworm. Twenty dominant-codominant trees were selected in each plot. Branch samples were collected both from the top third and from the middle third of their crowns. Needle loss and defoliation estimates were based on rating of the shoots under consideration in percentage loss or defoliation classes. Radial growth was measured at breast height.
\end{abstract}

One starting supposition was that estimating the respective needle loss percentages for the different age classes of shoots, being only taken into account those shoots apparently not yet affected by the natural needle fall, would give values approximately equal to those of the annual rates of budworm defoliation for the corresponding years. The use of such a method, we call retrospective estimation of the defoliation, was theoretically made possible by the conjunction of the following factors : (i) the fact that budworm larvae feed on the current-year needles and do not noticeably damage the old foliage even during outbreaks (fig. 1); (ii) the high life span, sometimes reaching at least 15 years, of the silver fir needles; and (iii) the monocyclism of silver fir, which allows us to accurately date the shoots. Two retrospective estimations of the budworm defoliations were successively made during winter 1985-1986 then in June 1987. The respective values they provided were compared between them, with those obtained the previous years by estimating current budworm defoliation a little after larval feeding period was completed, and also with old field observations (fig. 2). From these comparisons it appeared that the retrospectively estimated defoliation values gave an accurate picture of the variations in budworm damage intensity during the 10 previous years (fig. 3) and were not very different, at least for the 6 more recent years, from the actual budworm defoliation values. It is a result of great interest for its * Adresse actuelle : Département de la Santé des Forêts, Echelon technique inter-régional Nord-Ouest, 93, rue
de Curambourg, 45400 Fleury-les-Aubrais. 
possible applications and which most certainly makes $C$. murinana a rather exceptional case among forest defoliators. Both the natural needle fall and the action of other defoliation-causing factors are the cause of a certain error but the risk it should be important seems slight.

Whether or not budworm defoliation influences terminal growth of branches could not be determined from the collected data. Length growth reductions were observed the years of severe defoliation (fig. 4) but they could apparently be equally explained by the climatic conditions (i.e. drought) of the previous year. Severe defoliations had no noticeable effect upon the length of the shoots produced the following year.

Radial growth (fig. 5) was strongly influenced, but with a time lag of one year, by the severe budworm defoliation that occurred in one of the plots during the years 1979 and 1980. It was not noticeably influenced by defoliations about $50 \mathrm{p} .100$, perhaps because it was measured at breast height. Radial growth patterns in the trees showed several other growth reductions but nearly all of them coincided with an abnormally dry spring or summer. Sensibility of radial tree growth to many factors, among which climate, makes difficult the use of such a criterion for evidence of the past budworm outbreaks, at least when these outbreaks only lasted a short time.

Key words : Choristoneura murinana, Abies alba, population dynamics, defoliation, length growth, radial growth.

\section{Résumé}

Les taux annuels successifs de défoliation d'un peuplement d'Abies alba Mill. par C. murinana ont été rétrospectivement estimés, à deux reprises, par les taux de perte d'aiguilles des différentes classes d'âge de pousses, évalués sur les seules pousses apparemment non encore atteintes par la chute naturelle des aiguilles. Les valeurs fournies par les deux estimations ont été comparées entre elles, à celles obtenues les années précédentes en mesurant la défoliation courante peu après la fin de la période d'alimentation larvaire, enfin à d'anciennes observations de terrain. Cette comparaison a montré que la méthode utilisée permet de reconstituer a posteriori, sans grand risque d'erreur grave, semble-t-il, l'histoire de la défoliation par $C$. murinana sur environ 8-10 ans.

Les données recueillies n'ont pas permis de déterminer si la croissance en longueur des branches est ou non influencée par la défoliation : des croissances anormalement faibles ont été observées les années de sévère défoliation, mais elles peuvent tout aussi bien s'expliquer, apparemment, par les conditions climatiques (sécheresse) de l'année précédente. Les défoliations n'ont pas eu d'effet perceptible sur la longueur des pousses produites l'année suivante.

La croissance radiale des arbres a été fortement influencée, mais avec un an de retard, par une succession de deux années de très sévère défoliation; elle ne paraît pas l'avoir été par des défoliations de l'ordre de 50 p. 100 . Sa sensibilité à de nombreux facteurs (notamment au climat) rend malaisée l'utilisation d'un tel critère pour la détection des pullulations passées de la tordeuse, tout au moins lorsque ces pullulations ont été de brève durée.

Mots clés: Choristoneura murinana, Abies alba, dynamique population, défoliation, croissance longitudinale, croissance radiale.

\section{Introduction}

Lorsqu'un insecte forestier réputé inoffensif ou n'ayant jamais attiré l'attention se révèle brusquement commettre des dégâts importants par leur intensité ou par l'ampleur des surfaces atteintes, nombre de questions se posent, touchant par exemple à la nouveauté (réelle ? apparente ?) des pullulations observées, à leur devenir ou aux 
risques encourus par les peuplements encore épargnés; mais il est généralement impossible de leur apporter d'emblée ne serait-ce qu'un début de réponse, faute de connaissances sur le passé du ravageur.

Une telle situation se présente actuellement dans le sud de la France pour la tordeuse Choristoneura murinana (Hb.). Celle-ci, qui a pour hôte habituel le sapin pectiné, Abies alba Mill., et dont les dégâts sur cette essence sont classiques dans le centre et l'est de l'Europe (EIdmanN, 1949 ; PatočKa, 1960), était demeurée jusqu'à ces dernières années presque totalement inconnue en France (CoRNic et al., 1986). La découverte, en 1980, d'une pullulation de l'insecte dans une sapinière du département de l'Ardèche (LEMPÉRIÈre et al., 1984 ; CoRNIC et al., 1986), puis les résultats, pour la plupart encore inédits (voir toutefois CoRnIC et al., 1986 \& FABRE et al., 1987), d'une enquête par piégeage sexuel, conduisent aujourd'hui à la considérer comme un ennemi important, au moins potentiellement, des peuplements de sapin pectiné des Alpes et du Massif Central : l'espèce est présente un peu partout dans ces deux régions, une douzaine de foyers de pullulation y ont été mis en évidence.

La biologie générale de $C$. murinana est relativement bien connue (Franz, 1940 ; PatoČKa, 1960 ; Bogenschütz, 1978). L'insecte est univoltin. Les oufs sont déposés en été sur le feuillage de l'hôte et se développent en 10-15 jours. Il y a 6 stades larvaires. Les L1, qui ne s'alimentent pas, recherchent un abri (anfractuosité de l'écorce, etc.), puis y tissent un hibernaculum dans lequel elles muent. Les L2 passent l'automne et l'hiver à l'état de diapause dans cet hibernaculum. Au printemps, elles gagnent les bourgeons en cours de débourrement, y pénètrent et commencent alors à s'alimenter. Les stades larvaires L3 ou L4 à L6 consomment le feuillage épanoui de l'année. La période d'alimentation larvaire prend fin en juin-juillet.

Trouver des indicateurs qui permettraient de reconstituer, même très approximativement, les variations passées des niveaux de population du ravageur, tant dans les peuplements fortement infestés que dans ceux où sa densité est encore faible, serait d'un intérêt capital pour comprendre la situation actuellement observée dans le sud de la France et tenter de répondre aux diverses interrogations qu'elle suscite, dont celles ci-dessus évoquées.

L'objectif de l'étude ici présentée était de déterminer dans quelle mesure les dégâts commis par la tordeuse (destruction du feuillage) et certains des dommages qui en résultent pour les arbres (réduction de la croissance) pourraient fournir les informations recherchées.

\section{Méthodes}

\subsection{Les indicateurs potentiels étudiés}

Trois indicateurs potentiels des niveaux de population passés de la tordeuse ont été mis à l'épreuve :

- la perte d'aiguilles des pousses,

- l'accroissement longitudinal annuel des branches,

- l'accroissement radial annuel des arbres. 
Le choix des deux derniers allait quasiment de soi, car il est bien connu que les dégâts des insectes défoliateurs, lorsqu'ils sont importants, ont pour effet de diminuer la croissance des arbres (voir, entre autres, la synthèse déjà un peu ancienne mais toujours très utile de Kulman, 1971). L'analyse de cernes a par suite été assez souvent utilisée pour déceler et dater les pullulations passées de certains de ces ravageurs, par exemple celles de la tordeuse Choristoneura fumiferana (Clem.) (BlaIs, 1962, 1965, 1981).

La prise en considération, a priori assez surprenante, de la perte d'aiguilles des pousses a découlé, quant à elle, de certaines particularités de la biologie de la tordeuse et de son hôte, qui sont les suivantes :

a. Les chenilles de C. murinana, dans la nature, s'alimentent exclusivement sur le feuillage de l'année (Franz, 1940), même en cas de surpopulation et de famine (PAToČKA, 1960). Cette dernière affirmation demanderait peut-être à être légèrement nuancée : des L6 (stade de très loin le plus gros consommateur de feuillage), prélevées jeunes et que nous avons maintenues confinées, au laboratoire, sur du feuillage de l'année précédente, ont en effet fini par s'y attaquer, mais à un très faible degré (environ 2,5 aiguilles détruites en moyenne par chenille ; observations portant sur 92 individus, dont 46 p. 100 seulement sont parvenus au stade nymphal). Dans la nature, toutefois, lorsque la nourriture vient à manquer, les larves âgées se laissent tomber vers les strates inférieures. A supposer même que, devenues affamées, elles s'attaquent parfois au vieux feuillage, ces dégâts se limiteront donc aux plus basses branches et aux régénérations. Ils seront, de toute façon, négligeables à l'échelle de l'ensemble du peuplement.

b. La longévité des aiguilles du sapin pectiné est élevée : 15 ans au moins chez certaines des branches que nous avons examinées.

c. Le sapin pectiné est rigoureusement monocyclique ; en particulier, nous n'avons jamais observé de repousses d'été ou d'arrière-saison après une destruction totale du feuillage de l'année par la tordeuse. Les divers entre-nœuds des branches sont par suite, en règle générale, aisément datables ; ceci se révèle cependant parfois impossible pour les rameaux épicormiques ou pour certaines branches ayant acquis une ramification très buissonnante en raison d'accidents de croissance (quelquefois dus à la tordeuse).

Il résulte de ces faits que les dégâts commis une année par $C$. murinana demeurent longtemps visibles et datables. La figure 1 montre un cas extrême de "trou » de défoliation dû à l'insecte et qui aurait été encore bien visible une demi-douzaine d'années plus tard. Dans le peuplement où a été prélevé le rameau présenté, la population de l'insecte a été si importante en 1986 qu'elle y a détruit la presque totalité du feuillage de l'année, mais on remarquera que les aiguilles plus âgées du rameau ont été épargnées.

Il semblait donc que l'évaluation, sur des branches échantillons, des taux respectifs de perte d'aiguilles des différentes classes d'âge de pousses, à la condition de la limiter aux pousses non encore affectées par la chute naturelle du feuillage, pouvait fournir des valeurs approximativement exactes des taux annuels successifs de défoliation de ces branches par la tordeuse. C'est ce que nous avons voulu vérifier, en comparant, pour un même peuplement, les résultats obtenus avec cette méthode $(=$ estimation rétrospective de la défoliation) et ceux d'estimations antérieures de la défoliation courante, réalisées annuellement peu après la fin de la période d'alimentation larvaire (= estimation immédiate de la défoliation). Parallèlement, les arbres échantillonnés ont fait 


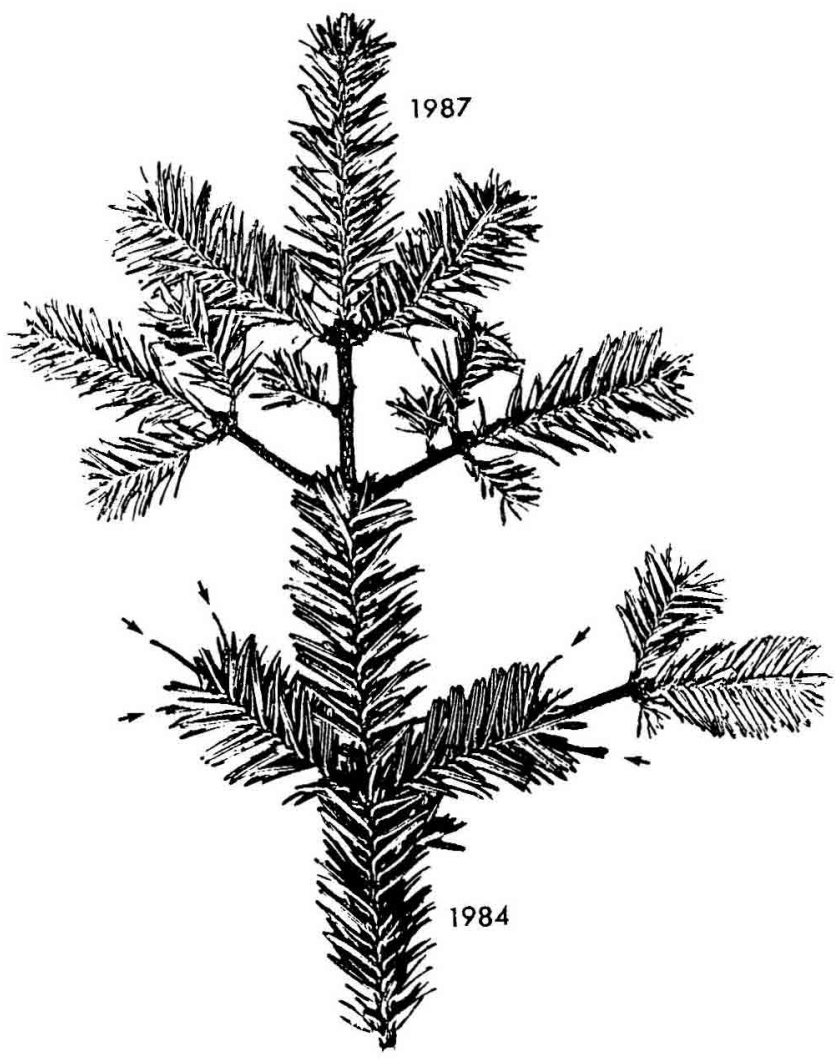

Fig. 1

Rameau d'Abies alba (4 années de pousses) prélevé en octobre 1987 dans une sapinière infestée par

C. murinana. La défolitation par la torảeuse a été nuile en 1994 et cn 1985 , totaie en 1986 (certaines pousses, indiquées par des flèches, étant même tuées par l'insecte), presque nulle en 1987.

Branch of Abies alba composed of 4 shoot years and cut in October 1987 in a stand infested by C. murinana. Budworm defoliation was nil in 1984 and in 1985, total with killing of some shoots (indicated with arrows) in 1986, almost nil in 1987. Note that in 1986 the old needles were not at all attacked despite the very high density of the larvae.

l'objet de mesures d'accroissement radial (du tronc) et longitudinal (des branches), dans le but de mettre en évidence d'éventuelles liaisons entre ces deux paramètres et la sévérité de la défoliation.

\subsection{Situation et caractéristiques des arbres échantillonnés}

Les observations ont été réalisées dans la sapinière ardéchoise (forêt de Combe Noire, Saint-Julien-Vocance) où a été découverte une très forte pullulation de la tordeuse en 1980. La dynamique de la population de l'insecte y est suivie depuis 1983 


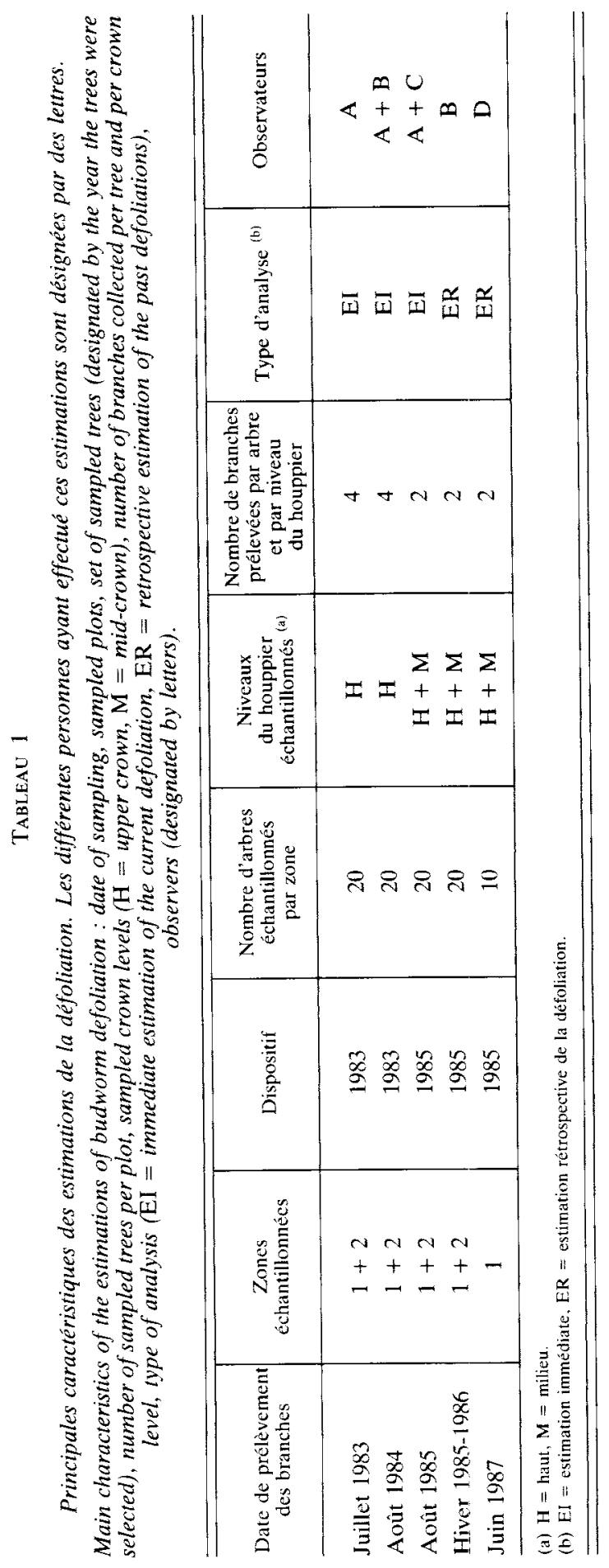


(CoRnic et al., 1986 et 1987 ; GÉraud et al., 1987). Deux zones d'étude y sont distinguées: l'une, dite zone 1, s'étage entre 850 et $950 \mathrm{~m}$ d'altitude et correspond au secteur le plus sévèrement infesté en 1980 ; l'autre, dite zone 2, lui succède entre 950 et $1100 \mathrm{~m}$ d'altitude. Dans les deux cas, le peuplement est une futaie naturelle adulte de sapin pectiné, presque pure et de structure irrégulière.

Au printemps 1983, 20 arbres dominants ou codominants, distants d'une vingtaine de mètres, ont été choisis dans chaque zone ; ceux de la zone 1 étaient hauts de 16 à $25 \mathrm{~m}$, ceux de la zone 2 de 19 à $30 \mathrm{~m}$ (Géraud et al., 1987). En 1985, ces arbres, qui avaient fait l'objet de plusieurs prélèvements de branches, ont été remplacés chacun par un voisin, choisi pour ses caractéristiques approximativement identiques. Les deux séries successives d'arbres seront respectivement dénommées dispositif 1983 et dispositif 1985.

\subsection{Estimations immédiates de la défoliation courante (tabl. 1)}

Ces estimations ont été effectuées en 1983, 1984 et 1985, parallèlement au dénombrement des pontes (Géraud et al., 1987), sur des extrémités de branches longues de $1 \mathrm{~m}$, prélevées en fin juillet ou début août, dès la fin du vol des adultes, selon le protocole suivant :

- en 1983 et 1984 : prélèvement, sur chacun des 40 arbres du dispositif 1983 , de 4 extrémités prises dans le tiers supérieur des houppiers ;

- en 1985: prélèvement, sur chacun des 40 arbres du dispositif 1985, de 2 extrémités prises dans le tiers supérieur des houppiers et de 2 extrémités prises dans leur tiers médian.

Les 2 ou 4 extrémités constituant chaque unité d'échantillonnage ont été récoltées au sein d'un même verticille ou sur des verticilles voisins, si possible selon 2 directions opposées ou selon 2 axes orthogonaux. Leurs pousses de l'année ont été dénombrées et le taux de défoliation de chacune évalué, 6 classes de défoliation étant distinguées : 0 , $0-20,20-40,40-60,60-80$ et 80-100 p. 100 en 1983 et $1984 ; 0,0-25,25-50,50-75,75-100$ et 100 p. 100 en 1985 (cette échelle de défoliation, dont les valeurs seuils sont $0,1 / 4$, $1 / 2,3 / 4$ et 1 , a été adoptée à partir de 1985 parce que plus "parlante » et donc d'un emploi plus aisé).

\subsection{Estimations rétrospectives des défoliations passées (tabl. 1)}

Au cours de l'hiver 1985-1986, 4 extrémités de branches longues de 1,50 m, dont 2 prises dans le tiers supérieur du houppier et 2 dans son tiers médian, ont été prélevées selon le même principe sur chacun des 40 arbres du dispositif 1985 . Chacune a ensuite fait l'objet des opérations suivantes: sélection de rameaux latéraux feuillés, répartis tout au long de la branche; datation des pousses composant ces rameaux et estimation du taux de perte d'aiguilles de chacune, l'échelle utilisée étant celle de pas 25 p. 100. Seules ont été notées les pousses pour lesquelles la chute naturelle des aiguilles paraissait ne pas être de nature, par son ampleur, à fausser les résultats. Cette appréciation, éviciemment très subjective, s'est basée à la fois sur la façon dont le taux de perte d'aiguilles évoluait chronologiquement le long des successions d'entre-nœuds, étant admis que l'intensité de la chute naturelle des aiguilles ne peut décroître quand on remonte dans le temps, et sur l'aspect général de la branche. Le taux de perte 
d'aiguilles a été estimé comme si celles récemment tombées, reconnaissables à la couleur claire des cicatrices foliaires, étaient encore présentes.

En juin 1987, 10 des arbres du dispositif 1985 de la zone 1 ont fait l'objet des mêmes observations, mais seules les pousses des années 1982 à 1985 ont été examinées.

\subsection{Mesure des allongements annuels des branches}

La longueur de chacun des entre-nieuds de l'axe des branches prélevées au cours de l'hiver 1985-1986 a été mesuré au $1 / 2 \mathrm{~cm}$ près. En cas d'interruption de l'axe d'origine, par suite de la destruction du bourgeon terminal, les mesures ont exclusivement porté, soit sur cet axe, soit sur le rameau faisant fonction d'axe de substitution. Aucune mesure n'a été faite sur les branches d'aspect trop buissonnant.

\subsection{Mesure des accroissements radiaux annuels des arbres}

En novembre 1985, une carotte a été prélevée à la tarière dans chacun des 40 arbres du dispositif 1985 . Les sondages ont été effectués à $1,30 \mathrm{~m}$ environ du sol, sur la face amont des arbres, jusqu'au coeur de ceux-ci. Les cernes de chaque carotte ont été lus à la loupe binoculaire, leur largeur étant mesurée à $0,04 \mathrm{~mm}$ près.

Ces mesures ont montré que les arbres de la zone 1 étaient plus àgés d'une trentaine d'années que ceux de la zone 2 (âges moyens respectifs à $1,30 \mathrm{~m}: 111$ ans et 80 ans).

En janvier 1986, des mesures identiques ont été effectuées sur 7 sapins dominants ou codominants de la forêt communale du Monestier (Ardèche), située à $3,5 \mathrm{~km}$ de la forêt de Combe Noire. Ces arbres étaient répartis dans une parcelle de caractéristiques assez semblables à celles de la zone I mais où $C$. murinana, bien que présent. paraît n'avoir jamais pullulé ces dernières années.

\subsection{Données manquantes}

De nombreuses données sont manquantes (ou inexistantes), pour les raisons suivantes: variabilité de l'âge des branches, variabilité de l'année en-deçà de laquelle il n'a plus été possible d'estimer rétrospectivement la défoliation, présence de branches trop buissonnantes, ou à axe incomplet, ou encore en mauvais état de conservation le jour de l'examen et par suite inutilisables pour l'estimation de la défoliation. De ce fait, les données analysées sont souvent inférieures en nombre à celles théoriquement disponibles d'après le tableau 1 .

\subsection{Expression des valeurs moyennes de la défoliation ei des accroissements annuels}

Les valeurs moyennes prises chaque année par les trois variables ont été calculées séparément pour chaque zone et pour chaque niveau des houppiers.

Le taux de défoliation d'une branche est la moyenne des taux de défoliation de ses pousses (calculée à partir des valeurs médianes des classes de défoliation). Celui d’un ensemble d'arbres sera exprimé par la moyenne par arbre des moyennes, pour chacun. des taux de défoliation des branches. 
De même, l'allongement annuel moyen des branches pour un ensemble d'arbres sera exprimé par la moyenne par arbre des moyennes, pour chacun, des allongements annuels des branches.

Enfin, l'accroissement radial annuel moyen d'un ensemble d'arbres sera évidemment exprimé par la moyenne par arbre des valeurs prises par la variable.

Les écarts entre taux de défoliation seront toujours donnés en valeur absolue.

\section{Résultats}

\subsection{Comparaison des estimations rétrospectives et immédiates de la défoliation}

Les données utilisées sont : les valeurs estimées immédiatement des défoliations de 1983, 1984 et 1985 ; les valeurs estimées rétrospectivement, au cours de l'hiver 19851986, des défoliations des années 1982 à 1985 ; les valeurs estimées rétrospectivement, en 1987, de ces mêmes défoliations.

Pour chaque combinaison zone-année n'ont été retenues que les données relatives aux arbres communs à la fois aux diverses estimations de la défoliation et aux deux niveaux des houppiers, de façon à rendre les échantillons pris en compte tous similaires (tabl. 2).

La figure 2 compare, par zone, niveau des houppiers et année de production des pousses, les résultats des trois estimations de la défoliation. On constate les faits suivants :

- Années 1982, 1983, 1984 : L'estimation immédiate et les deux estimations rétrospectives de la défoliation ont donné des résultats ne différant que de façon négligeable (écart maximal : 6,6 p. 100). La légère augmentation en 1987, par rapport à 1985-1986, de la défoliation estimée des pousses $1982(3,6$ à 6,6 p. 100 selon le niveau des houppiers) pourrait correspondre à une chute naturelle d'aiguilles. La diminution tout aussi légère $(5,0$ p. 100) en 1985-1986, par rapport à 1983 , de celle des pousses 1983 des arbres de la zone 2 pourrait, quant à elle, résulter d'une faible surévaluation de leur défoliation lors de son estimation immédiate : en 1983, en effet, un grand nombre des branches prélevées dans la zone 2 (28 branches, soit 7 arbres complets) n'ont pu être analysées car elles s'étaient mal conservées et perdaient leurs aiguilles ; peut-être certaines de celles qui ont servi à estimer la défoliation avaient-elles, par conséquent, commencé à perdre quelques aiguilles sans que nous y ayons pris garde.

- Année 1985: En ce qui concerne la zone 2, l'estimation immédiate de la défoliation et celle réalisée au cours de l'hiver suivant ont donné des résultats identiques (écart de 0,5 à 1,5 p. 100 selon le niveau des houppiers). En ce qui concerne la zone 1, au contraire, on note une nette évolution dans le temps de la valeur estimée de la défoliation, évolution qui n'est pas la même pour les deux niveaux des houppiers : la défoliation estimée des branches hautes s'accroît (de 9,5 p. 100) entre l'estimation immédiate (année $n$ ) et la première estimation rétrospective (année $n+0,5$ ) puis ne varie plus jusqu'à la seconde (année $n+2$ ), celle des branches médianes ne varie pas entre l'estimation immédiate et la première estimation rétrospective puis s'accroît (de 8,3 p. 100) entre celle-ci et la seconde. Par ailleurs, tant l'estimation immédiate que la 


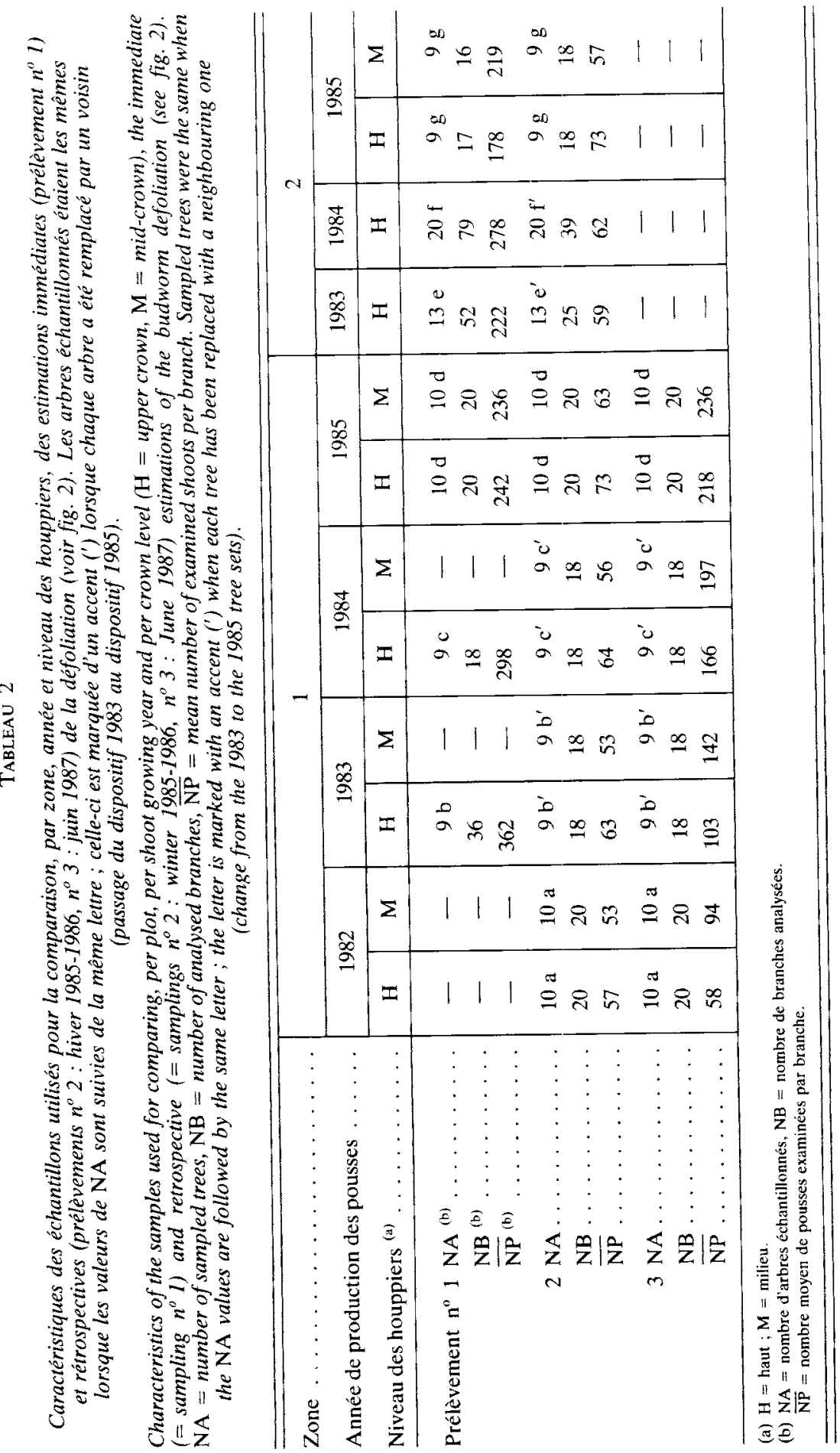



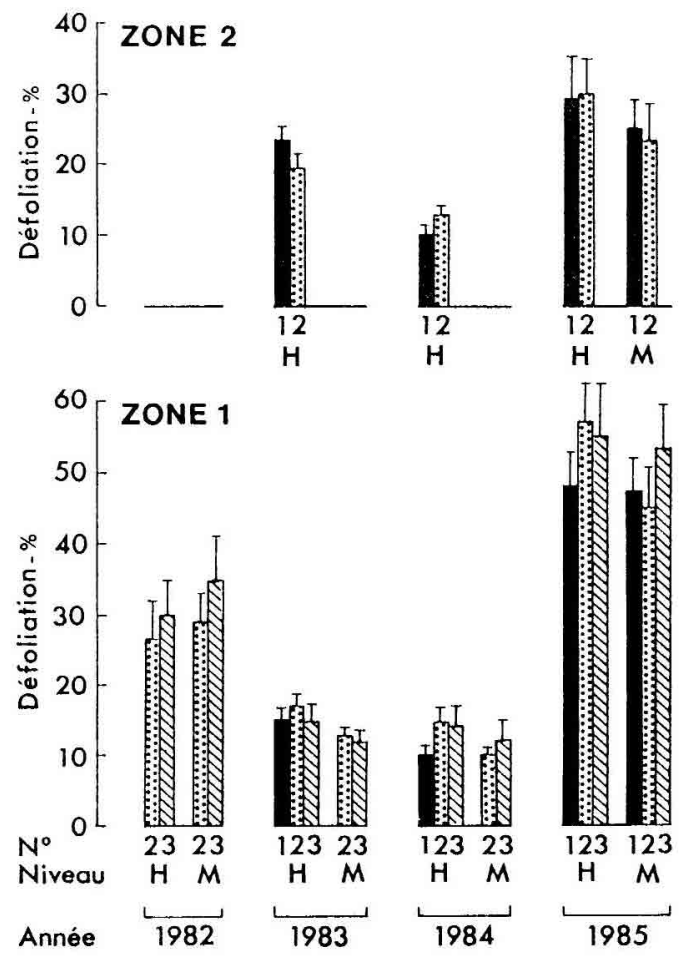

FIG. 2

Comparaison, par zone, par niveau des houppiers $(\mathrm{H}=$ haut, $\mathrm{M}=$ médian $)$, et pour chacune des annèes 1982 à 1985, des valeurs moyennes de la défoliation respectivement estimées : immédiatement $\left(n^{\circ} 1\right)$; rétrospectivement au cours de l'hiver 1985-1986 ( $\left.n^{o} 2\right)$; rétrospectivement en juin 1987 $\left(n^{\circ} 3\right)$. Les traits verticaux représentent l'erreur standard.

Comparison, per plot, per crown level $(\mathrm{H}=$ upper crown, $\mathrm{M}=$ mid-crown $)$, and for each of the years 1982 to 1985, of the mean values of budworm defoliation respectively estimated: immediatly $\left(=\right.$ sampling $\left.n^{\circ} 1\right)$, retrospectively during winter 1985-1986 (= sampling $\left.n^{\circ} 2\right)$, and retrospectively in June 1987 (= sampling $\left.n^{\circ} 3\right)$. Bars indicate standard errors of the mean.

seconde estimation rétrospective ont fourni des valeurs semblables de la défoliation pour les deux niveaux des houppiers. Il semble donc que les pousses 1985 ont subi dans la zone 1, quelque temps après leur attaque par la tordeuse, une perte d'aiguilles, de même intensité pour les deux niveaux des houppiers, mais plus précoce pour les branches hautes. Le facteur responsable de ce phénomène demeure inconnu : chute anticipée d'aiguilles d'origine physiologique? maladie cryptogamique? attaque d'insectes? En tout cas, l'ampleur du phénomène est demeurée limitée puisque les écarts entre les valeurs extrêmes déduites des diverses estimations de la défoliation n'ont pas atteint 10 p. 100.

Au total, les estimations rétrospectives ont donc donné des valeurs de la défoliation ne différant guère, ou que de façon tout à fait tolérable, de celles estimées immédiatement jusqu'à 4 années plus tôt (cas des pousses 1983 examinées en 1987), et 
même, très vraisemblablement, de celles qui l'auraient été 5 années plus tôt (cas des pousses 1982 examinées en 1987). Cette conclusion est d'autant plus intéressante que les diverses estimations de la défoliation ont été faites par des observateurs variés (tabl. 1).

Il faut enfin signaler qu'en 1986, les dégâts commis par la tordeuse dans la zone 1 ont été extrêmement sévères (95-98 p. 100 de défoliation moyenne selon le niveau des houppiers), à un point tel que les axes de nombreuses jeunes pousses ont été euxmêmes rongés, que les cimes de certains arbres se montraient enveloppées d'un dense réseau de fils soyeux abandonnés par les larves et que les dommages subis par les jeunes régénérations furent presque aussi importants que ceux infligés aux arbres. Cette situation de surpopulation, confinant à la famine, n'a eu aucun effet perceptible sur les pousses âgées, ce que montre la comparaison des valeurs de leur défoliation estimées respectivement avant et après le printemps 1986. Les valeurs plus élevées, en 1987, de la défoliation des pousses 1982 des branches hautes et médianes et de celle des pousses 1985 des branches médianes ne peuvent lui être attribuées, dans le premier cas parce qu'on n'observe un tel phénomène ni pour les pousses 1983 ni pour les pousses 1984, pourtant plus récentes, dans le second parce que l'évolution, semblable mais plus précoce, de la défoliation estimée des pousses 1985 des branches hautes indique qu'il faut en chercher l'explication ailleurs. Ces observations démontrent clairement que les larves de $C$. murinana, dans les conditions naturelles et même en période de pullulation, n'attaquent pas de façon perceptible le feuillage âgé des grands arbres.

\subsection{Reconstitution de l'histoire de la défoliation dans la forêt de Combe Noire}

Les résultats de l'estimation rétrospective de la défoliation réalisée au cours de l'hiver 1985-1986 sont présentés fig. 3. L'examen du tableau 3 montre que les données, pour chaque zone, ne sont pas exactement comparables entre niveaux des houppiers; elles le sont encore moins entre années, le nombre des arbres étudiés diminuant au fur et à mesure qu'on remonte dans le temps. Ceci ne prête toutefois guère à conséquence. Lorsqu'on restreint, en effet, l'analyse à des sous-échantillons tels que les mêmes arbres soient alors pris en compte pour les deux niveaux des houppiers ou d'année en année, la forme des courbes obtenues et leurs positions respectives ne s'en trouvent pratiquement pas modifiées.

Les données permettent de remonter jusqu'en 1976, soit sur 10 ans. Les faits rapportés dans le chapitre précédent ont montré que les valeurs estimées rétrospectivement des défoliations respectives de $1985,1984,1983$ et même très vraisemblablement 1982 ne diffèrent que peu de celles estimées ou qui auraient été estimées immédiatement : la forme des courbes déduites de ces dernières serait exactement la même que celle des courbes de la figure 3. Qu'en est-il alors pour les années précédentes ?

Le foyer de pullulation de Combe Noire a été découvert en 1980. Les dégâts étaient tellement importants qu'ils étaient visibles de loin, mais seul le bas de la forêt, correspondant approximativement à la zone 1, a été jugé, à l'époque, sévèrement endommagé. Les résultats de l'estimation rétrospective concordent tout à fait avec ces observations puisqu'ils indiquent que la défoliation des pousses 1980 a été quasi-totale dans la zone 1, mais bien moindre, et par suite passant facilement inaperçue, compte tenu de la hauteur des arbres, dans la zone 2, qui lui succède en altitude. 


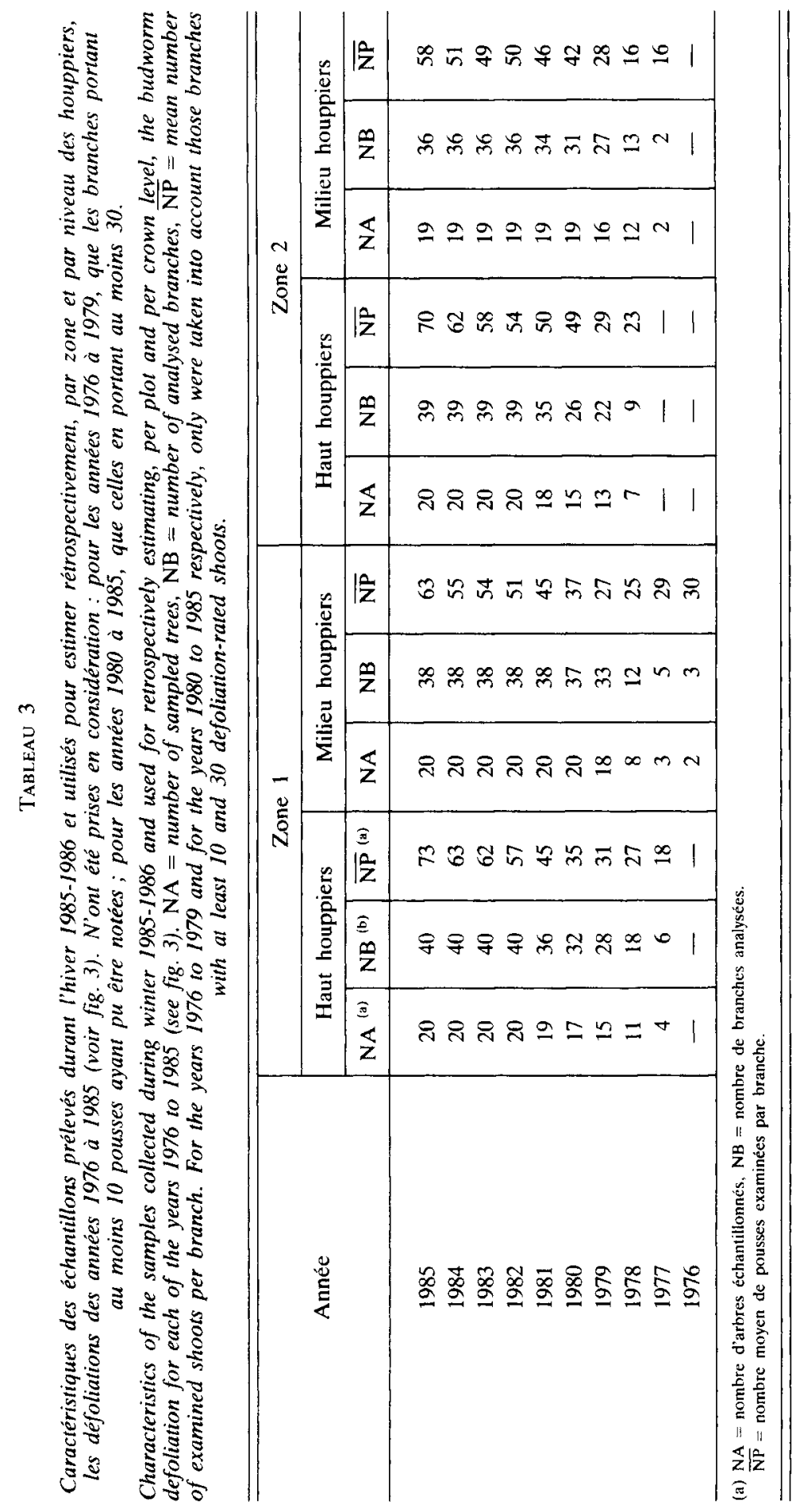



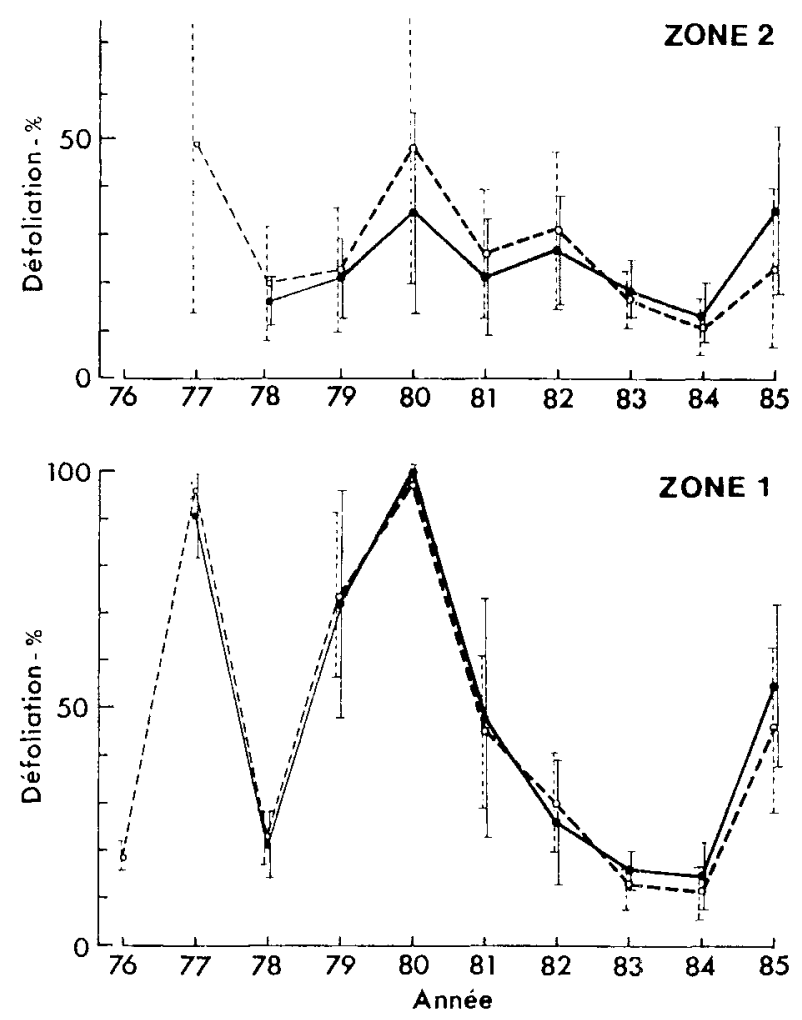

FIG. 3

Valeurs moyennes par zone, estimées rétrospectivement durant l'hiver 1985-1986, des défoliations respectives des branches hautes (traits pleins) et des branches médianes (tiretés) pour les années 1976 à 1985 ; en traits fins : données approximatives (faibles effectifs des échantillons d'arbres, branches et pousses). Les traits verticaux représentent l'écart-type.

Retrospectively estimated mean values for the years 1976 to 1985 of the budworm defoliation in each plot, based on analysis of the branches collected during winter 1985-1986; solid lines: upper crown branches; broken lines: mid-crown branches; fine lines : data from small-sized tree, branch and shoot samples. Bars indicate standard deviations.

Antérieurement à 1979, et surtout à 1978, la taille des échantillons (nombre d'arbres, de branches, de pousses) diminue tellement (tabl. 3) qu'on est a priori tenté de ne considérer les résultats correspondants qu'avec suspicion, d'autant qu'il y a tout lieu de craindre, à leur sujet, que l'erreur résultant de la chute naturelle des aiguilles ne devienne importante. Néanmoins, plusieurs observations relatives à la zone 1 conduisent à réviser ce jugement. Tout d'abord, l'évolution estimée de la défoliation entre 1978 et 1980 y est tout à fait comparable, y compris quantitativement, à celle observée entre 1984 et 1986 (année où, rappelons-le, le taux de défoliation a quasiment atteint 100 p. 100). D'autre part, nous savons, par un bûcheron, que certains au moins des arbres de cette zone ont été sévèrement infestés en 1977, ce qui concorde parfaitement avec les taux de défoliation très élevés déduits de lanalyse des branches hautes et 
médianes. On doit de plus noter que ces branches, au total, étaient au nombre de 11 (tabl. 3) et préciser qu'elles étaient réparties entre 6 arbres (dont un seul commun aux deux catégories de branches) : les données relatives à l'année 1977, lorsqu'elles sont considérées globalement, se rapportent donc à un échantillon déjà assez important. II ne fait ainsi guère de doute que la défoliation moyenne des arbres de la zone 1 a atteint une valeur élevée en 1977, peut-être inférieure à son estimation de 90-95 p. 100, mais en tout cas contrastant fortement avec celle de 1978.

La défoliation de 1976 dans la zone 1 , estimée à 19 p. 100, a été déduite de l'analyse des branches basses de 2 arbres seulement (tabl. 3). En 1977, la valeur estimée de la défoliation moyenne de ces mêmes branches était de 96 p. 100. Le taux de défoliation de certaines au moins des branches de la zone 1 s'est donc indubitablement accrû fortement de 1976 à 1977. Il est par suite très vraisemblable que la défoliation moyenne des arbres de cette zone était moindre la première de ces deux années.

Au total, l'évolution de l'action défoliatrice passée de la tordeuse décrite par les courbes de la figure 3 s'avère donc correspondre fidèlement à son évolution réelle, et même n'en différer quantitativement qu'assez peu, au moins pour la période 1980-1985.

Enfin, on notera que les valeurs estimées respectives de la défoliation pour les deux niveaux étudiés des houppiers ont toujours été quasiment identiques, à quelques exceptions près, pour lesquelles l'écart est faible (au plus 13,5 p. 100).

\subsection{Allongements annuels moyens des branches}

Les branches étudiées sont celles prélevées au cours de l'hiver 1985-1986. Pour chacune des quatre combinaisons zone-niveau des houppiers, la composition de l'échantillon varie fortement selon l'année considérée. Aussi a-t-on choisi d'analyser les résultats par périodes de temps successives, chacune correspondant à un échantillon de composition constante et ayant une année en commun avec la suivante (tabl. 4).

Les variations, au cours du temps, de l'allongement annuel des branches sont présentées figure 4 , où elles sont comparées à celles de la défoliation. L'allure générale des courbes est la même pour les branches hautes et pour les branches médianes. Elle est également à peu près la même pour les deux zones (sauf de 1981 à 1982).

Dans chacune, la croissance des branches a fortement diminué en 1971, en 1977 et en 1979-1980. Dans les deux derniers cas, cette croissance réduite a coïncidé avec une augmentation du taux de défoliation, très importante dans la zone 1. La similitude, pour cette zone, entre les courbes de croissance et la courbc (inversée) de défoliation est telle que, considérée seule, elle ne laisserait quasiment aucun doute sur l'existence d'une relation de cause à effet entre les deux phénomènes. Ceci amènerait alors à supposer que l'insecte avait déjà commis de sévères dégâts en 1971 .

De 1978 à 1980, toutefois, le ralentissement de la croissance des branches a été, en valeur absolue, aussi important dans la zone 2 que dans la zone 1 , alors que la première a été beaucoup moins fortement infestée que la seconde.

D'autre part, les années 1970, 1976, 1978 et 1979, qui ont précédé les réductions de croissance observées, se sont toutes caractérisées par un printemps ou un été momentanément ou continuellement très secs (tab. 5 et fig. 4 ; à noter que les effets sur la réserve en eau du sol de certains des déficits de précipitations mentionnés dans le 


\section{TABLFAU 4}

C'aractéristiques des échantillons utilisés pour la mesure de l'allongement annuel moyen des branches (voir fig. 4); à chacune des périodes distinguées correspond un échantillon particulier,

$$
\text { de composition constante. }
$$

Characteristics of the samples used for measuring the mean annual length increment of the branches (see fig. 4). NA = number of sampled trees. NB = number of measured branches. To cach of the distinguished time periods corresponds a particular samiple, of constant composition.

\begin{tabular}{|c|c|c|c|c|c|c|}
\hline \multirow{2}{*}{ Niveau des houppiers } & \multicolumn{3}{|c|}{ Zone 1} & \multicolumn{3}{|c|}{ Zone 2} \\
\hline & Période & $\mathrm{NA}^{\text {(a) }}$ & $N B^{(a)}$ & Période & NA & NB \\
\hline \multirow[t]{10}{*}{ Haut } & $1969-1970$ & 8 & 11 & $1972-1973$ & 7 & 11 \\
\hline & $1970-1971$ & 10 & 17 & $1973-1974$ & 7 & 13 \\
\hline & $1971-1972$ & 12 & 21 & $1974-1975$ & 9 & 16 \\
\hline & $1972-1973$ & 14 & 26 & $1975-1976$ & 12 & 20 \\
\hline & 1973-1974 & 17 & 30 & $1976-1977$ & 18 & 28 \\
\hline & $1974-1975$ & 19 & 35 & $1977-1978$ & 18 & 32 \\
\hline & $1975-1978$ & 20 & 40 & $1978-1979$ & 18 & 31 \\
\hline & $1978-1981$ & 20 & 39 & $1979-1981$ & 19 & 34 \\
\hline & $1981-1983$ & 20 & 38 & $1981-1984$ & 18 & 33 \\
\hline & $1983-1985$ & 20 & 37 & $1984-1985$ & 18 & 32 \\
\hline \multirow[t]{9}{*}{ Milieu } & $1966-1967$ & 8 & 12 & $1969-1970$ & 8 & 10 \\
\hline & $1967-1968$ & 10 & 14 & $1970-1971$ & 9 & 14 \\
\hline & $1968-1970$ & 12 & 17 & $1971-1972$ & 10 & 15 \\
\hline & $1970-1972$ & 14 & 19 & $1972-1973$ & 13 & 20 \\
\hline & $1972-1975$ & 13 & 18 & $1973-1974$ & 13 & 23 \\
\hline & $1975-1977$ & 13 & 19 & $1974-1975$ & 16 & 28 \\
\hline & $1977-1980$ & 12 & 18 & $1975-1976$ & 17 & 30 \\
\hline & $1980-1985$ & 11 & 17 & $1976-1981$ & 17 & 31 \\
\hline & & & & $1981-1985$ & 17 & 30 \\
\hline
\end{tabular}

(a) NA = nombre ditrbres chantillennes, NB = nombre de branches inesurecs

tableau se sont sans doute prolongés bien au-delà du retour à une pluviométrie normale).

Bien qu'un examen détaillé des données climatiques conduise parfois à s’interroger sur les relations entre croissance des branches et pluviométrie de l'année précédente, cet ensemble de faits montre que les données recueillies ne permettent pas de répondre à la question de savoir si la défoliation influence ou non cette croissance. L'examen de la figure 4 fournit néanmoins une information intéressante : les sévères défoliations de 1977 et de 1980 n'ont pas eu d'effet marqué sur la longueur des pousses produites l'année suivante.

\subsection{Accroissements radiaux annuels moyens}

Les variations, au cours de la période 1954-1985, des croissances radiales respectives des 20 arbres de la zone 1 , des 20 arbres de la zone 2 et des 7 arbres de la forêt du Monestier sont présentées figure 5, comparativement à celles de la défoliation. 

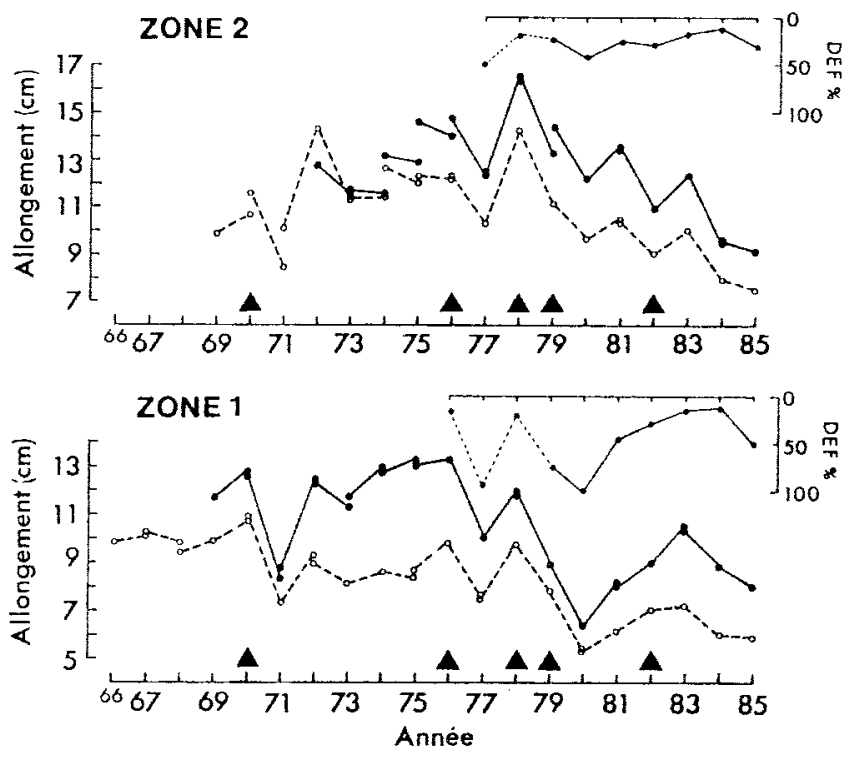

FIG, 4

Allongements annuels moyens, par zone, des branches hautes (traits pleins) et des branches médianes (tiretés), comparés à la défoliation moyenne des deux niveaux des houppiers. Les années à printemps ou été anormalement secs (tabl. 5) sont signalées par des triangles noirs.

Mean annual length increments for each plot of the upper crown branches (solid lines) and of the mid-crown branches (broken lines) compared with the mean defoliation of the two crown levels.

Solid triangles indicate years with abnormally dry spring or summer (table 5).

\section{Tableau 5}

Principaux épisodes de sécheresse ayant pu influencer la croissance longitudinale et radiale des arbres au cours de la période 1954-1985. D'après les données du poste "La Louvesc " (Ardèche) de la Météorologie Nationale, distant de $7,5 \mathrm{~km}$ du site de Combe Noire et situé à une altitude comparable (l $050 \mathrm{~m}$ ).

Main water deficits that could have influenced length or radial growth of the trees during 1954-1985.

Data from the meteorological station "La Louvesc" (Ardeche) situated $7.5 \mathrm{~km}$ away from the study site, at a similar elevation $(1050 \mathrm{~m}) . \mathrm{P}=$ pluviometry,

as a percentage of its mean value for the $1957-1986$ period.

\begin{tabular}{|c|c|c|}
\hline Année(s) & Epoque & $\begin{array}{c}\text { Pluviométrie } P \text {, en pourcentage de sa valeur moyenne } \\
\text { pour la période } 1957-1986\end{array}$ \\
\hline 1956 & Avril-Juin & $P=54$ p. 100 (mais juillet : $P=240$ p. 100 ) \\
\hline $1961-1962$ & Décembre-Août & $P=63$ p. 100 \\
\hline 1970 & Juin-Septembre & $P=57$ p. 100 \\
\hline $1975-1976$ & Octobre-Juin & $\begin{array}{l}P=55 \text { p. } 100 \text { (mais juillet, août et septembre } 1976 \\
\text { avec } P>100 \text { p. } 100 \text { ) }\end{array}$ \\
\hline 1978 & Juin-Septembre & $\begin{array}{l}P=55 \text { p. } 100 \text { (mais juillet, août avec } \\
P=85 \text { p. } 100,91 \text { p. } 100 \text { ) }\end{array}$ \\
\hline 1979 & Juin-Septembre & $\begin{array}{l}P=66 \text { p. } 100 \text { (mais juin, août avec } \\
P=99 \text { p. } 100,105 \text { p. } 100)\end{array}$ \\
\hline $1981-1982$ & Octobre-Juin & $P=62$ p. 100 \\
\hline
\end{tabular}


Les trois profils de croissance sont remarquablement semblables, à deux exceptions près : pour la période 1983-1984 d'une part (la cause en demeure inconnue), et surtout pour la période 1979-1982, durant laquelle l'accroissement radial annuel moyen des arbres de la zone 1 a continuellement varié en sens inverse de ceux des arbres de la zone 2 et du Monestier. La quasi-égalité des accroissements radiaux dans la zone 1 et au Monestier au cours des années antérieures (1971-1979) rend cette dernière anomalie encore plus frappante. La comparaison des trois profils de croissance, entre eux, d'une part, et avec les courbes de défoliation, d'autre part, démontre clairement que la sévère défoliation des arbres de la zone 1 en 1979 (70 p. 100) puis en 1980 (100 p. 100) a eu un fort effet dépressif sur leur croissance, tout d'abord en 1980 puis à nouveau, de façon beaucoup plus marquée, en 1981 (l'accroissement radial moyen de 1981 par exemple, par rapport à sa valeur de 1979, a augmenté de respectivement 35 p. 100 et 40 p. 100 dans la zone 2 et au Monestier, qui servent ici de témoins, mais diminué de 25 p. 100 dans la zone 1). La réponse des arbres de la zone 1 à une forte défoliation n'a donc pas été immédiate mais différée d'un an. La défcliation seulement modérée (estimée à 35-50 p. 100 selon le niveau des houppiers) des arbres de la zone 2 en 1980 n'a eu, quant à elles, aucun effet visible sur leurs accroissements radiaux.

La défoliation apparemment très élevée des arbres de la zone 1 en 1977 n'a pas eu non plus d'effet perceptible, immédiat ou différé, sur leur croissance radiale. Ceci suggère que l'intensité moyenne de cette défoliation a été assez nettement inférieure à sa valeur estimée, déduite, rappelons-le, de l'examen d'un assez petit nombre de branches. Mais il se peut aussi que les valeurs estimées de cette intensité pour les années 1976 à 1978 soient approximativement exactes, auquel cas l'absence de réaction des arbres pourrait s'expliquer par le caractère très fugace de la pullulation.

A plusieurs reprises $(1956,1962,1970,1974,1976)$, la croissance radiale des arbres s'est ralentie plus ou moins fortement et de façon synchrone dans les trois peuplements. Ce phénomène a généralement coïncidé avec un déficit prononcé des précipitations de printemps ou d'été (tabl. 5 et fig. 5). Une relation de cause à effet paraît indubitable dans tous les cas, même si les sécheresses, il est vrai intermittentes, des étés 1978 et 1979 n'ont pas ou guère influencé de façon perceptible les accroissements radiaux. La réduction de croissance particulièrement spectaculaire de 1956 pourrait également avoir été provoquée, ou accentuée, par les températures très exceptionnellement basses qu'a connue la France au cours du mois de février de cette année. Il n'est pas possible pour autant d'affirmer que la tordeuse n'a joué aucun rôle dans ces diverses pertes de croissance, mais son action, si elle s'est faite sentir, ne peut être alors distinguée de celle du climat.

Le ralentissement de la croissance radiale des arbres observé en 1973 puis à nouveau en 1974 demeure, quant à lui, sans cause connue, car aucune explication du phénomène ne se dégage de l'examen des données climatiques (température, précipitations). On pourrait, dans ces conditions, penser aux effets d'une pullulation de la tordeuse, mais le fait que les trois peuplements ont été affectés simultanément rend une telle hypothèse plutôt douteuse.

A la forte réduction de la croissance des arbres de la zone 2 et du Monestier en 1982, résultant apparemment d'un climat anormalement sec, s'oppose une légère tendance à la reprise pour ceux de la zone 1. En l'absence de sécheresse, l'accroissement radial de ces arbres aurait donc sans doute retrouvé dès 1982 un niveau équivalent, voire peut-être nettement supérieur, à celui de 1980. Il est par suite vraisemblable, d'une part que leur défoliation à près de 50 p. 100 en 1981 n'a guère influencé leur 


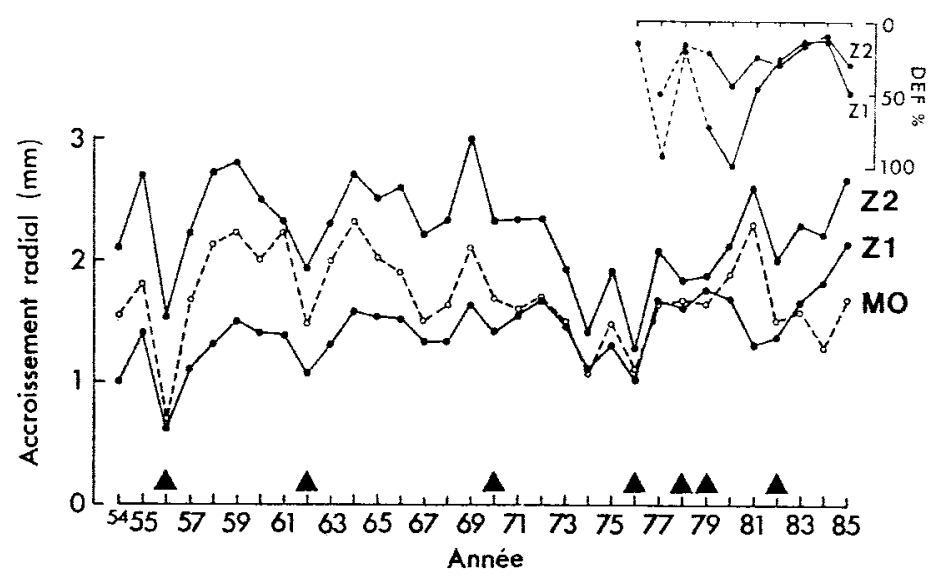

FIG. 5

Accroissements radiaux annuels moyens à $1,30 \mathrm{~m}$ des 20 arbres de la zone 1 (Z1), des 20 arbres de la zone 2 (Z2) et des 7 arbres de la forêt du Monestier (MO). Comparaison avec la défoliation moyenne des deux niveaux des houppiers dans chacune des deux zones. Les années à printemps ou été anormalement secs (tabl. 5) sont signalées par des triangles noirs; de plus, février 1956 a été très anormalement froid.

Mean annual radial increments at breast height for the 20 trees of the plot 1 ( $\mathrm{Z1})$, for the 20 trees of the plot 2 (Z2) and for the 7 trees of the Monestier Forest (MO), a silver fir stand lying $3.5 \mathrm{~km}$ away and which does not seem to have been damaged by C. murinana these last years. Comparison with the mean defoliation of the two crown levels in each of the two plots. Solid triangles indicate years with abnormally dry spring or summer (table 5); moreover,

February 1956 was very abnormally cold.

croissance radiale l'année suivante, tout comme la défoliation, d'intensité voisine (3550 p. 100), des arbres de la zone 2 en 1980 était restée sans effet sur celle de ces derniers, d'autre part que leur défoliation successivement sévère puis extrême en 19791980 n'a eu qu'un effet fugace : il n'a commencé à se faire sentir (ainsi que noté plus haut) qu'en 1980 et était probablement déjà en grande partie annulé en 1982.

\section{Discussion et conclusions}

\subsection{Estimation rétrospective de la défoliation}

MacLeAn \& Morgan (1981) ont montré, dans le cas de branches de sapin baumier (Abies balsamea (L.) Mill.) attaquées par Choristoneura fumiferana (Clem.), que l'appréciation visuelle de la défoliation des pousses pratiquée, ainsi que nous l'avons fait, en utilisant une échelle de notation à 6 classes donne des résultats satisfaisants : en moyenne, la valeur estimée de la défoliation était quasiment égale à sa valeur réelle ; l'appréciation visuelle surestimait très légèrement la défoliation moyenne des pousses dont les aiguilles étaient détruites à plus de 80 p. 100 , elle sous-estimait celle des pousses moins attaquées. 
D’un autre côté, les observations rapportées dans les chapitres précédents indiquent que le facteur "observateur " n'a pas eu d'effet perceptible sur les résultats des estimations de la défoliation. Elles démontrent également, dans un autre ordre d'idées, que les larves de $C$. murinana, dans la nature, épargnent totalement le feuillage âgé des grands arbres, ou du moins ne l'endommagent pas de façon appréciable, même en période de surpopulation.

Dans la mesure où l'action défoliatrice d'autres facteurs ne vient pas interférer avec celle de la tordeuse et où l'on parvient à faire la part de la chute naturelle des aiguilles, l'appréciation visuelle du taux moyen de perte d'aiguilles de n'importe quelle classe d'âge de pousses d'un échantillon de branches de sapin pectiné fournit donc, pour l'année considérée, une valeur de la défoliation de ces branches par l'insecte qui est certainement très voisine de ce qu'était sa valeur réelle. C'est ce qu'avaient déjà entrevu Franz (1940) \& PatočKa (1963). Mais ceux-ci n'ont pas vraiment analysé les fondements de cette méthode, n'ont pas cherché à en éprouver la fiabilité et sont loin d'en avoir tiré tout le parti possible.

L'analyse des branches prélevées à Combe Noire au cours de l'hiver 1985-1986 a permis de reconstituer, de façon hypothétique tout d'abord, l'évolution dans le temps de la défoliation au cours des 10 années précédentes (1976-1985). La comparaison de ces données, d'une part avec les résultats d'estimations immédiates de la défoliation réalisées les années précédentes, d'autre part avec ceux d'une autre estimation rétrospective, enfin avec d'anciennes observations de terrain, prouve de façon quasiment indubitable : (a) que les profils de défoliation ainsi obtenus restituaient fidèlement la forme des fluctuations passées de l'intensité des dégâts commis par l'insecte, et (b) que les valeurs estimées rétrospectivement de cette intensité ne différaient que peu, au moins pour les 6 dernières années considérées (1980-1985), cle ce qu'étaient ou auraient été leurs valeurs estimées immédiatement.

L'estimation rétrospective de la défoliation a néanmoins des limites, qui sont de diverses natures:

- Le nombre des années pouvant être reconstituées est étroitement déterminé par la durée de vie des aiguilles du sapin pectiné. Celle-ci paraît n'avoir été que très rarement étudiée. Les informations données par Gourbière $(1981,1982)$ et par Gourbière \& Corman (1985) sont basées sur l'examen d'un échantillon beaucoup trop réduit pour être généralisables, mais elles semblent bien démontrer que la longévité moyenne des aiguilles croît assez fortement du haut vers le bas des houppiers. Cette longévité varie certainement assez largement d'un arbre à l'autre - ceci est manifeste dans la forêt de Combe Noire, bien que la durée de vie moyenne des aiguilles ne puisse y être évaluée avec précision du fait des attaques de la tordeuse - mais aussi selon le peuplement. Dans l'un de ceux que nous avons étudiés, les axes des branches du tiers supérieur des houppiers portaient une douzaine d'années complètes ou presque d'aiguilles, et nous avons observé, dans un autre peuplement, mais sur un arbre de lisière il est vrai, des branches basses dont l'axe en portait au moins 15 .

- La précision des résultats est, quant à elle, fonction de la façon dont la chute naturelle des aiguilles évolue de l'apex vers la base des branches et des rameaux. Gourbière (1981, 1982) \& Gourbière \& Corman (1985) donnent également quelques indications sur ce point, mais ici aussi existe sans doute une certaine variabilité entre arbres et entre peuplements. En tout cas, on doit évidemment s'attendre à ce que l'erreur résultant de la confusion entre chute naturelle des aiguilles et défoliation par la tordeuse augmente quand on remonte dans le temps. Mais il faut également attirer 
l'attention sur le fait suivant: lors de l'analyse des branches, nous avons admis que la perte d'aiguilles par chute naturelle ne peut décroître lorsqu'on passe d'une classe d'âge de pousses à la précédente ; or, ceci demanderait à être vérifié, car la longévité naturelle des aiguilles varie peut-être parfois selon l'année de formation des pousses.

- Les divers facteurs autres que la tordeuse susceptibles d'exercer une action "défoliatrice", qu'il s'agisse d'insectes, de maladies ou de facteurs physiologiques, constituent une autre source potentielle d'erreur. En ce qui concerne au moins les insectes, le risque est toutefois faible, car très rares sont ceux qui parviennent à commettre des dégâts importants : outre $C$. murinana, on ne peut guère citer que la tordeuse Zeiraphera rufimitrana (H.-S.), apparemment nettement moins nuisible; il arrive néanmoins que l'action cumulée des autres espèces composant le complexe des Lépidoptères défoliateurs du sapin pectiné soit loin d'être négligeable (PATočKA, 1960).

En dépit de ces limites, l'estimation rétrospective de la défoliation, comme le montre l'exemple de la forêt de Combe Noire, est une méthode d'un intérêt manifestement considérable puisqu'elle permet de reconstituer a posteriori, sans grand risque de se tromper gravement, les fluctuations de l'intensité des dégâts commis par l'insecte et par suite, au moins dans une certaine mesure, celles de son niveau de population sur un laps de temps qui doit être généralement de l'ordre de 8-10 ans. Ceci est tout à fait remarquable et fait de $C$. murinana un cas assurément assez exceptionnel chez les défoliateurs forestiers, car tenant à la conjonction de certaines particularités de la biologie de l'insecte (régime alimentaire) et de son hôte (monocyclisme, longévité des feuilles) certainement très rarement réunies. La méthode, par exemple, ne pourrait sans doute pas s'appliquer à certains au moins des Choristoneura nord-américains des Abietoidea. Parmi leurs hôtes (voir Harvey, 1985), en effet, figurent des essences (Pseudotsuga menziesii (Mirb.) Franco, Picea spp.) à tendance polycyclique, produisant en particulier des repousses après de fortes défoliations (Volney, 1985), ce qui rend sans doute difficile voire impossible une datation précise des entre-nœuds. De plus, trois espèces au moins, $C$. fumiferana (Clem.), C. occidentalis Free. et $C$. biennis Free., sont capables d'endommager le feuillage âgé (voir par exemple HARRIS, 1964 ; TALERICO, 1984 ; FELLIN, 1985).

\subsection{Croissance longitudinale des branches}

De nombreux auteurs ont montré que les dégâts commis par les tordeuses nordaméricaines des Abietoidea $C$. fumiferana, $C$. occidentalis et $C$. retiniana (Wlsm.) (= viridis Free.) provoquent, lorsqu'ils sont sévères, des pertes importantes de croissance en hauteur des arbres (BATZER, 1973 ; BASKenville \& MACLEAN, 1979 ; BousFIELD, 1980 ; FerRell, 1980 ; ThOMSON \& VAN Sickle, 1980 ; VAN Sickle et al., 1983 ; VAN SICKLE, 1985 ; etc.). Mais les observations qu'ils rapportent ne font pas la part entre l'effet de la défoliation sur la longueur de la pousse terminale et, d'un autre côté, les pertes de croissance résultant de la destruction de bourgeons terminaux par l'insecte et des dessèchements de cimes provoqués par l'action défoliatrice de celui-ci.

Nos propres observations ne permettent pas de déterminer si les dégâts commis par C. murinana influencent ou non la croissance en longueur des branches car les croissances anormalement faibles enregistrées les années de forte défoliation peuvent tout aussi bien s'expliquer, semble-t-il, par les conditions climatiques (sécheresse) de l'année précédente. Cette influence des conditions environnementales de l'année $n-1$ sur la longueur des pousses produites l'année $n$ est classique chez les essences dont les 
pousses sont entièrement préformées dans les bourgeons avant l'hiver, ce qui est le cas pour le sapin pectiné (G. Aussenac, comm. pers.) ; elle se montre alors généralement bien supérieure à celle des conditions de l'année $n$, du moins lorsque la croissance en longueur des pousses s'achève tôt en saison (voir par exemple Becker, 1977 ; Pollard \& Logan, 1977 ; Kramer \& Kozlowski, 1979 ; Kozlowski, 1982 ; Guyon, 1986). Cette dernière caractéristique est présentée par le sapin pectiné, dont les pousses, par exemple, ne s'allongent plus guère dès le mois de juin ou le début du mois de juillet dans la région de Nancy (Debazac, 1965, 1967 ; Aussenac, 1975 a et b).

On aurait pu s'attendre, dans ces conditions, à ce que la défoliation influence la croissance des branches de cette essence, d'une part l'année même des dégâts, puisque ceux-ci affectent les jeunes pousses durant leur phase d'allongement, d'autre part, et peut-être même surtout, l'année suivante, en agissant sur la mise en place des primordiums dans les bourgeons. Nos résultats ne mettent toutefois en évidence aucun effet différé sensible de la défoliation sur la longueur des pousses.

La question de savoir si les variations dans le temps des allongements annuels des branches peuvent fournir des informations sur l'action défoliatrice passée de la tordeuse reste donc ouverte. Des recherches sont en cours pour tenter d'y répondre. En tout état de cause, un tel critère serait sans doute d'un emploi assez malaisé car nombreux sont les facteurs susceptibles d'avoir une action sur la croissance en longueur des branches. A l'effet du climat s'ajoute, par exemple, celui de la floraison. Morris (1951) \& BlaIs (1952) ont montré que l'intensité de celle-ci influence fortement la longueur des pousses chez Abies balsamea. Des observations récentes, demandant à être complétées, indiquent qu'il en va de même chez le sapin pectiné (Du Merle \& Cornic, données non publiées).

\subsection{Croissance radiale des arbres}

La croissance radiale à $1,30 \mathrm{~m}$ des arbres de la zone 1 a été fortement influencée, mais avec une année de retard, par les très sévères défoliations de 1979-1980, tandis que celle des arbres de la zone 2 ne paraît pas l'avoir été par leur défoliation, estimée à seulement $35-50$ p. 100 , de 1980 . Lors des pullulations des Choristoneura nordaméricains se développant sur Abies, Picea ou Pseudotsuga, on observe également un décalage d'une à plusieurs années entre défoliation et réduction de la croissance radiale de l'hôte lorsque celle-ci est mesurée, comme nous l'avons fait, à hauteur d'homme (Blais, 1958 ; Maclean, 1981 ; Alfaro et al., 1982 ; Archambault, 1984 ; Van SICKLE, 1985). Mais cette réduction est à la fois plus précoce et plus intense lorsqu'elle est mesurée au niveau du houppier (Williams, 1967 ; Piene, 1980 ; Thomson \& Van Sickle, 1980 ; Maclean, 1981, 1985 ; Van Sickle, 1985), du moins lorsqu'elle est exprimée en valeur absolue, car, exprimée en pourcentage de la croissance potentielle, elle ne variait pas de façon marquée du bas vers le haut des arbres analysés par Alfaro et al. (1985). Peut-être, par conséquent, une réponse des arbres de la zone 2 à la défoliation seulement modérée de 1980 aurait-elle été apparente si les accroissements radiaux de ces arbres avaient été mesurés au niveau du houppier.

En tout cas, comme ceci était prévisible, les variations entre années des accroissements radiaux s'avèrent susceptibles de nous fournir de précieuses informations, au moins sur l'existence passée de fortes pullulations de C. murinana. Toutefois, l'exemple de la forêt de Combe Noire montre également, d'une part que des défoliations très sévères n'ont peut-être pas d'effet visible sur la croissance radiale, ou du moins sur 
celle mesurée à $1,30 \mathrm{~m}$, lorsqu'elles sont très fugaces (cas hypothétique de l'année 1977 pour la zone 1), d'autre part que l'instabilité du climat peut rendre difficile l'interprétation des résultats. Les liaisons observées entre croissance radiale et conditions climatiques de la même année sont classiques chez les arbres forestiers, lesquels se montrent parfois également sensibles au climat de l'année précédente (Aussenac, 1973, 1978 ; Becker, 1977 ; Kramer \& Kozlowski, 1979 ; KozlowsKi, 1982 ; etc.). L'influence, moins connue bien qu'importante, de la floraison et de la fructification des arbres sur leur croissance radiale (MoRRIs, 1951 ; EIs et al., 1965) doit être aussi prise en compte et complique encore le problème. En fait, celui-ci ne se laisse aisément résoudre que lorsque les pullulations, comme c'est le cas pour celles de C. fumiferana au Canada, persistent de nombreuses années : les profils de croissance radiale des arbres présentent alors, en effet, de profondes et larges dépressions, dont l'interprétation, comme le montrent les graphiques présentés par BLAIS $(1962,1965,1981)$, ne laisse généralement pas place au doute.

\section{Remerciements}

Cette étude a été réalisée dans le cadre d'une convention de recherche passée entre l'INRA et le Syndicat départemental d'Equipement de l'Ardèche. Nous remercions Sylvie Brunet et JeanFrançois Coknic, qui ont participé à la collecte des données, ainsi que Gilbert AussenaC, pour ses conseils lors de la rédaction du manuscrit.

Reçu le 5 janvier 1988.

Accepté le 9 mai 1988.

\section{Références bibliographiques}

Alfaro R.I., Van Sickle G.A., Thomson A.J., Wegnitz E., 1982. Tree mortality and radial growth losses caused by the western spruce budworm in a Douglas-fir stand in British Columbia. Can. J. For. Res., 12, 780-787.

Alfaro R.I., Thomson A.J., Van Sickle G.A., 1985. Ouantification of Douglas-fir losses caused by the western spruce budworm defoliation using stem analysis. Can. J. For. Res., 15, 5-9.

Archambault L., 1984. Impact de la défoliation causée par la tordeuse des bourgeons de l'épinette sur la croissance en volume de trois sapinières. Rev. Rech. Serv. Can. For., 4, n"1, 4-6.

Aussenac G., 1973. Climat, microclimat et production ligneuse. Ann. Sci. For., 30, 239-258.

Aussenac G., 1975 a. Etude de la croissance en hauteur chez quelques résineux, ellet de la température. Ann. Sci. For., 32, 1-16.

Aussenac G., 1975 b. Couverts forestiers et facteurs du climat: leurs interactions, conséquences écophysiologiques chez quelques résineux. Thèse Docteur ès Sciences naturelles, Univ. Nancy I, $241 \mathrm{p}$.

Aussenac G., 1978. La sécheresse de 1976 : influence des déficits hydriques sur la croissance des arbres forestiers. Rev. For. Fr., 30, 103-114.

Baskerville G.L., MACLEAN D.A., 1979. Budworm-caused mortality and 20-year recovery in immature balsam fir stands. Can. For. Serv., Inf. Rep. M-X-102, 23 p. 
Batzer H.O., 1973. Net effect of spruce budworm defoliation on mortality and growth of balsam fir. J. For., 71, 34-37.

Beckfr M., 1977. Contribution à l'étude de la transpiration et de l'adaptation à la sécheresse des jeunes plants résineux. Exemple de 3 sapins du pourtour méditerranéen (Abies alba, $A$. nordmanniana, A. numidica). Ann. Sci. For. 34, 137-158.

BLAIS J.R., 1952. The relationship of the spruce budworm (Choristoneura fumiferana, Clem.) to the flowering condition of balsam fir (Abies balsamea (L.) Mill.). Can. J. Zool, 30, 1-29.

Bi.AIS J.R., 1958. Effects of defoliation hy spruce budworm (Choristoneura fumiferana $\mathrm{Clcm}$.) on radial growth at breast height of balsam fir (Abies balsamea (L.) Mill.) and white spruce (Picea glauca (Moench) Voss.). For. Chron., 34, 39-47.

BLAIS J.R., 1962. Collection and analysis of radial-growth data from trees for evidence of past spruce budworm outbreaks. For. Chron., 38, 474-484.

BLals J.R., 1965. Spruce budworm outbreaks in the past three centuries in the Laurentide Park. Quebec. Forest Sci., 11, 130-138.

BLAIS J.R., 1981. Recurrence of spruce budworm outbreaks for two hundred years in Western Quebec. For. Chron., 57, 273-275.

Bogenschütz H., 1978. Tortricidae. In Die Forstschädlinge Europas, Bd. III, W. Schwenke ed., Paul Parey, Hamburg und Berlin, 55-89.

Bousfiel. W.E., 1980. The effects of height growth loss on stands damaged by the western spruce budworm. U.S.D.A., For. Serv., Northern Region. State \& Private Forestry, Forest Insect and Disease Management, Missoula, Montana, Rept, 80-11, 3 p.

Cornic J.F., Géraud D., Du Merle P., 1986. Evolution de la pullulation de la tordeuse du sapin Choristoneura murinana Hb. (Lép., Tortricidae) dans la forêt de Combe Noire (Ardèche) et données préliminaires sur la situation du ravageur dans le sud-est de la France. Rev'. For. Fr., 38, $538-550$.

Cornic J.F., Gŕknud D.. Du Mrrle P., 1987. Observations sur la distribution spatiale et sur la mesure des populations pré-imaginales de la tordeuse du sapin, Choristoneura murinana $\mathrm{Hb}$. (Lép. Tortricidae). 2. Les populations larvaires et conclusion. Z. Ang. Ent., 103. 403-417.

Debazac E.F.. 1965. Observations sur le débourrement et la croissance en longueur de quelques espèces de sapins. Rev. For. Fr., 17, 120-130.

Dibazac E.F., 1967. Nouvelles observations sur le débourrement et la croissance en longueur de quelques espèces de sapins. Rev. For. Fr.. 19, 183-190.

Eidmann H., 1949. Verbreitung und Schadgebiete des Tannentricbwicklers Cacoecia murinama Hb. (Lep. Tortricidae). Anz. Schädlingsk, 22, 103-107.

Eis S., Garman E.H., Ebili. L.F., 1965. Relation between cone production and diameter increment of Douglas fir (Pseudotsuga menziesii (Mirb.) Franco), grand fir (Abies grandis (Dougl.) Lindl.), and western white pine (Pinus monticola Dougl.). Can. J. Bot., 43. 15531559.

Fabre J.P.. Du Merle P.. Chalon A., 1987. Un nouveau ravageur du cèdre en Europe Choristoneura murinana, la tordeuse du sapin. Rei'. For. Fr., 39

Fel.IIN D.G., 1985. Western budworm and its hosts. In Managing trees and stands susceptible to western spruce budworm, M.H. Brookes. J.J. Colbert, R.G Mitchell et R.W. Stark eds. U.S.D.A., For. Serv., Techn. Bull.. 1695. 7-14.

Ferrell. G.T., 1980. Growth of white firs defoliated by modoc budworm in Northeastern California. U.S.D.A., For. Serv. Pacific Southwest Forest and Range Exp. Stn., Res. Pap., PSW-153, 9 p.

FranZ J., 1940. Der Tannentricbwickler Cacoecia murinana $\mathrm{Hb}$. Beiträge zur Bionomic und Ökologic. Z. Ang. Ent.. 27, 345-407 + 585-620.

Géraud D., Cornic J.F., Du Mrrl. P.. 1987. Observations sur la distribution spatiale et sur la mesure des populations pré-imaginales de la tordeuse du sapin. Choristoneura murinana $\mathrm{Hb}$. (Lép., Tortricidae). 1. Exposé des méthodes. Les populations d'œufs. Z. Ang. Ent.. 103. 258278 .

Gourbièke F., 1981. Vie, sénescence et décomposition des aiguilles de sapin (Abies alba Mill.). I. - Méthodologie et premiers résultats. Acta Oecol-Oecol. Plant. 2. 223-232.

Gourbière F., 1982. Vie, sénescence et décomposition des aiguilles de sapin (Ahies alba Mill.) 2. Bilan annuel. Acta Oecol.-Oecol. Plamt. 3. 185-196. 
Gourbière F., Corman A., 1985. Vie, sénescence et décomposition des aiguilles de sapin (Abies alba Mill.). 5. - Répartition et variations verticales des aiguilles vivantes. Acta Oecol.-Oecol. Plant., 6, 193-209.

GUYon J.P., 1986. Influence du climat sur lexpression des composantes de la croissance en hauteur chez le pin noir d'Autriche (Pinus nigra Arn. ssp. nigricans). Ann. Sci. For., 43, $207-$ 226.

HarRis J.W.E., 1964. Sampling the larval stages of the two-year-cycle spruce budworm near Babine Lake, British Columbia. For. Chron., 40, 195-201.

Harvey G.T., 1985. The taxonomy of the coniferophagous Choristoneura (Lepidoptera : Tortricidae): a review. In Recent advances in spruce budworms research, Proceedings of the CANUSA Spruce Budworms Research Symposium, Bangor, Maine, September 16-20, 1984, C.J. Sanders, R.W. Stark, E.J. Mullins et J. Murphy eds., Can. For. Serv., Ottawa, 16-48.

Kozlowski T.T., 1982. Water supply and tree growth. Part I. Water deficits. Forestry Abstracts, 43, $\mathbf{n}^{0} 2,57-95$.

Kramer P.J., Koziowski T.T., 1979. Physiology of woody plants. Academic Press, New York, San Francisco, London, $811 \mathrm{p}$.

Kulman H.M.. 1971. Effects of insect defoliation on growth and mortality of trees. Ann. Rev. Ent., 16, 289-324

Lempérière G., Givors A., SABatier B.. 1984. Une infestation de Choristoneura murinana Hb., tordeuse du sapin, dans le nord de l'Ardèche. Rev. For. Fr., 36, 206-210.

MACLEAN D.A., 1981. Impact of defoliation by spruce budworm populations on radial and volume growth of balsam fir : a review of present knowledge. Mitt. Forst. Bundes-Versuchsanst, Wien, 142, 293-306.

MacLean D.A., 1985. Effects of spruce budworm outbreaks on forest growth and yield. In Recent advances in spruce budworms research, Proceedings of the CANUSA Spruce Budworms Research Symposium, Bangor, Maine, September 16-20, 1984, C.J. Sanders, R.W. Stark, E.J. Mullins et J. Murphy eds., Can. For. Serv., Ottawa, 148-175.

Maclean D.A., Morgan M.G., 1981. The use of phyllotaxis in estimating defoliation of individual balsam fir shoots. Can. For. Serv., Res. Notes, 1, 12-14.

Morris R.F., 1951. The effects of flowering on the foliage production and growth of balsam fir. For. Chron., 27, 40-57.

Patočka J., 1960. Die Tannenschmetterlinge der Slowakei mit Berücksichtigung der Fauna Mitteleuropas. Slowak. Akad. Wiss., Bratislava, 215 p.

Patočka J., 1963. Zur Frage der Beeinflussung der Tannentriebwicklergradation durch die Wetterbedingungen in der kritischen Zeitspanne. Z, ang. Ent., 51, 195-202.

Prene H., 1980. Effects of insect defoliation on growth and foliar nutrients of young balsam fir. Forest. Sci., 26, 665-673.

Pollard D.F.W., Logan K.T., 1977. The effects of light intensity, photoperiod, soil moisture potential, and temperature on bud morphogenesis in Picea species. Can. J. For. Res., 7, 415421 .

TALERico R.L., 1984. General biology of the spruce budworm and its hosts. In Managing the spruce budworm in Eastern North America, D.M. Schmitt, D.G. Grimble et J.L. Searcy eds., U.S.D.A., For. Serv., Agric. Handb., 620, 1-10.

Thomson A.J., VAN Sickle G.A., 1980. Estimation of tree growth losses caused by pest activity. Can. J. For. Res., 10, 176-182.

VAN Sickle G.A., 1985. Effects of infestations on trees and stands. In Managing trees and stands susceptible to western spruce budworm, M.H. Brookes, J.J. Colbert, R.G. Mitchell et R.W. Stark eds., U.S.D.A., For. Serv., Techn. Bull., 1695, 15-21.

VAN Sickle G.A., Alfaro R.I., Thomson A.J., 1983. Douglas-fir height growth affected by western spruce budworm. Can. J. For. Res., 13, 445-450.

Volney W.J.A., 1985. Comparative population biologies of North American spruce budworms. In Recent advances in spruce budworms research, Proceedings of the CANUSA Spruce Budworms Research Symposium, Bangor, Maine, September 16-20, 1984, C.J. Sanders, R.W. Stark, E.J. Mullins et J. Murphy eds., Can. For. Serv., Ottawa, 71-84.

Williams C.B., 1967. Spruce budworm damage symptoms related to radial growth of grand fir, Douglas-fir and Engelmann spruce. Forest Sci., 13, 274-285. 